\title{
eJRIEPS
}

Ejournal de la recherche sur l'intervention en éducation physique et sport

16 | 2009

Varia

\section{La mixité en Education Physique et Sportive : points de vue d'élèves du second degré}

\section{Vanessa Lentillon}

\section{(2) OpenEdition}

\section{Journals}

Édition électronique

URL : https://journals.openedition.org/ejrieps/6799

DOI : 10.4000/ejrieps.6799

ISSN : 2105-0821

Éditeur

ELLIADD

\section{Référence électronique}

Vanessa Lentillon, «La mixité en Education Physique et Sportive : points de vue d'élèves du second degré », eJRIEPS [En ligne], 16 | 2009, mis en ligne le 01 janvier 2009, consulté le 10 juillet 2021. URL : http://journals.openedition.org/ejrieps/6799; DOI : https://doi.org/10.4000/ejrieps.6799

\section{(c) (1)}

La revue eJRIEPS est mise à disposition selon les termes de la Creative Commons Attribution 4.0 International License. 
La mixité en Education Physique et Sportive : points de vue d'élèves du second degré

Vanessa Lentillon

Institut des Sciences du Sport et de l'Education Physique, Université de Lausanne, Suisse

Résumé

L'Education Physique et Sportive (EPS) est une discipline d'enseignement mixte à forte connotation masculine. L'objet de cette étude est de déceler le regard que portent les élèves du second degré sur la mixité en EPS. Des entretiens semi-directifs ont été réalisés auprès de 86 élèves et une analyse de contenu de type "logico-sémantique " a été menée. Les élèves sont globalement favorables à la mixité, surtout les lycéens et les élèves d'établissements favorisés. Les attitudes des filles et des garçons sont ambiguës et très stéréotypées. La mixité est appréciée surtout grâce à la présence des garçons, perçus comme " marrants ", " motivants " et "forts » en EPS. La domination du masculin en EPS est ancrée dans les perceptions des élèves.

À partir de loi Haby en 1975, la mixité scolaire est devenue obligatoire et elle s'est progressivement généralisée dans tous les établissements scolaires. Le but de cet article est de mettre au jour le regard que portent les élèves du second degré sur la mixité en Education Physique et Sportive (EPS). Les études antérieures se sont essentiellement centrées sur la mise en place effective de la mixité et ont analysé les inégalités intersexes persistantes à travers les contenus enseignés, les attitudes et interventions de l'enseignant, etc. Cet article comble un certain manque puisqu'il aborde la mixité du côté des élèves et analyse les différences de points de vue des élèves selon leur sexe, leur niveau de scolarité (collège / lycée) et le type d'établissement fréquenté (favorisé / défavorisé). Nous allons, dans un premier temps, voir les limites de la mixité scolaire et plus particulièrement en EPS. Ensuite, les travaux antérieurs sur le point de vue des élèves à l'égard de la mixité en EPS vont être abordés. Enfin, notre étude sera présentée.

\section{Cadre conceptuel}

1. 1. La mixité scolaire, un monde au «masculin neutre »

Les filles et les garçons à l'école vivent des expériences sociales n'offrant pas le même type d'opportunité de réalisation de soi. L'école apparaît comme un lieu de socialisation 
sexuée paradoxal : à la fois égalitaire et source de progrès social, elle participe également au maintien du système catégoriel asymétrique de sexe (Duru-Bellat 2002 ; Mosconi 1994 ; Zaidman 1995). Nicole Mosconi analyse la mixité comme la réalisation d'un monde au «masculin neutre » : « neutre » parce que la différence tend à y être déniée, mais « masculin » parce que le genre masculin y reste dominant. Qu'en est-il en EPS ?

L'EPS, s'appuyant sur le sport, est connotée « masculine ». Le sport en général est une activité qui, par son histoire et les représentations (l'affrontement, le défi et l'épreuve) reste profondément masculine (Connell 1995 ; Gilroy 1989 ; Naess 2001 ; Terret 2004). Alors que l'école et les textes officiels prônent l'équité en EPS, les classes d'éducation physique sont des lieux de production d'un discours qui police et renforce les idées dominantes de la masculinité (Cleuziou 2000 ; Cogérino 2005 ; Colwell 1999 ; Couchot-Schiex 2005 ; Flintoff 1994 ; Skelton 2002 ; Vertinsky 1992 ; Vigneron 2004). Cette valorisation du masculin en EPS se manifeste sous divers aspects : des activités connotées « masculines » plus nombreuses; des entrées dans l'activité « étrangères » pour les filles; des barèmes peu sensibles aux différences génétiques; des attentes et interventions stéréotypées des enseignants en faveur des garçons; une plus grande réussite des garçons ; des comportements et attitudes des élèves très stéréotypés.

Comme dans les autres disciplines d'enseignement, les garçons mettent en place en EPS des « stratégies d'accaparement de l'attention enseignante » (Mosconi 1994) notamment par la création d'incidents disciplinaires (Couchot-Schiex \& Trottin 2005 ; Lentillon \& Trottin 2005 ; Leroy et al. 1998). Les filles restent en retrait face aux comportements agressifs, compétitifs des garçons (Satina et al. 1998 ; Vertinsky 1992), face à leurs attitudes méprisantes, de supériorité (Dallaire \& Rail 1996 ; Griffin 1984 ; Rønholt 2002). La présence des garçons a des effets négatifs sur la participation des filles : ces dernières initient davantage d'interactions dans les classes non mixtes que dans les classes mixtes (MacDonald 1990).

\section{2. La mixité en EPS : qu'en pensent les élèves ?}

Des études antérieures permettent d'apporter quelques connaissances sur le point de vue des élèves à l'égard de la mixité en EPS. Les élèves semblent plutôt favorables à la mixité en EPS (Combaz 1991 ; Dallaire \& Rail 1996 ; Gagné, Martel \& Nadeau 2006). Les relations conflictuelles entre les élèves et le manque d'esprit sportif sont rarement évoqués par les élèves comme un problème en EPS (Coelho 2000). Selon Artus (1999), plus le niveau de classe augmente et plus les lycéens sont pour la mixité en EPS.

Plus que les filles, les garçons sont pour la mixité en EPS (Combaz 1991 ; Dallaire \& Rail 1996 ; Gagné et al. 2006 ; Wright 1996). Les garçons «bons » apprécient plus 
particulièrement la mixité afin de regarder les filles en tenue sportive et développer des relations personnelles avec ces dernières ; les garçons «pas bons » en EPS fondent leur préférence pour la mixité sur le fait de pouvoir jouer avec des personnes de niveau plus faible (Gagné et al. 2006). Les filles expriment davantage de réticences à l'égard de la mixité : elles sont plus nombreuses à souhaiter seulement « de temps en temps » que les cours soient mixtes en EPS (56 \% contre 44 \%) (Enquête du MEN, 1984-1985). Elles privilégient la mixité sous certaines conditions (Dallaire \& Rail 1996) : lorsque les sports d'équipe sont moins axés sur la compétition et plus sur la participation, l'interaction et le « fun »; si les garçons n'adoptent pas d'attitudes de supériorité vis-à-vis d'elles ; lorsque le jeu se déroule dans une atmosphère où le langage et les attitudes sexistes sont absents.

Notre étude permet de combler un manque puisqu'aucune étude antérieure n'a comparé le point de vue des élèves à l'égard de la mixité selon leur niveau de scolarité (collège / lycée) et le type d'établissement fréquenté (favorisé / défavorisé). Nous cherchons à déceler comment aujourd'hui les élèves d'établissements secondaires français vivent la relation à l'autre sexe en EPS et une comparaison fine des réponses des filles et des garçons, des collégiens et des lycéens, des élèves d'établissements favorisés et défavorisés a été menée.

Au regard des résultats des études antérieures, nous supposons que les élèves sont globalement satisfaits de la mixité en EPS et qu'elle est perçue plus positivement par les garçons que les filles. Nous supposons également que les collégiens et les élèves d'établissements défavorisés ont une attitude plus négative à l'égard de la mixité. Les relations à l'autre sexe sont plus difficiles pour ces élèves puisque les normes de genre sont plus rigides lors de la puberté (Galambos, Almeida \& Petersen 1990) et dans les milieux sociaux défavorisés (Bouchard \& Saint-Amand 1996).

\section{Méthodologie}

\section{1. Outil de recueil de données}

$\mathrm{Au}$ total, quarante-quatre entretiens semi-directifs ont été réalisés auprès d'élèves du second degré. Ces entretiens se sont déroulés la plupart du temps de façon collective, avec deux ou trois élèves volontaires pendant les heures libérées des élèves et dans un endroit calme (cf. Tableau 1). Ce choix d'interroger prioritairement les élèves par deux ou trois a été fait suite à une étude préalable (Lentillon \& Cogérino 2005). Les élèves, à deux ou à trois, s'expriment beaucoup plus, acquiescent les réponses de l'autre, les complètent, les nuancent ou les contestent. Dans neuf cas, un seul élève était disponible pour réaliser 
l'entretien. Ces entretiens individuels ont été pris en compte même si les données sont moins riches. Les entretiens ont été réalisés dans la majorité des cas en situation nonmixte (40 sur 44), les élèves étant souvent dans la cour de récréation en compagnie d'élèves de même sexe. Les entretiens ont tous été enregistrés et dactylographiés. Ils ont duré en moyenne 28 minutes et au total 20h 42 min d'entretiens ont été retranscrites.

Tableau I. Nombre d'élèves par entretien

\begin{tabular}{||l||l|l|l|l||}
\hline $\begin{array}{l}\text { Nombre d'élèves par } \\
\text { entretien }\end{array}$ & 1 élève & 2 élèves & 3 élèves & Total \\
\hline Nombre d'entretiens & 9 & 28 & 7 & 44 \\
\hline
\end{tabular}

La grille d'entretien a été réalisée suite à une revue de littérature sur les inégalités intersexes présentes en EPS et a été utilisée pour un travail plus large portant sur les injustices perçues par les élèves (Lentillon 2006). Nous nous centrons ici aux réponses des élèves à la question portant sur la mixité : «Préférez-vous être mélangés filles et garçons en EPS ou séparés ? Pourquoi ? ».

\section{2. Échantillon}

Au total, 86 élèves volontaires du second degré ont été interrogés (43 filles (F) / 43 garçons (G), 33 collégiens (Co) / 53 lycéens (Ly) de filières générale, technologique ou professionnelle). L'âge moyen des élèves est de 16.1 ans $(\mathrm{ET}=2.6)$ et suit la loi normale (K-S : N = 86, Dmax $\left.=0.14,{ }^{* *}\right)$. Les élèves interviewés appartiennent pour 46 d'entre eux à deux établissements " défavorisés » (Défav) et les 40 autres à deux établissements «favorisés 》 (Fav). Le classement en établissements « favorisés » et « défavorisés »a été réalisé en fonction de trois indicateurs: les catégories socioprofessionnelles des familles des élèves fréquentant l'établissement, le taux de réussite des élèves au diplôme national du brevet (pour le collège) et du baccalauréat (pour le lycée) et le pourcentage d'élèves d'origine étrangère fréquentant l'établissement (cf. Tableau 2). II n'y a pas de différence significative entre les sexes en ce qui concerne le niveau de scolarité (collège / lycée) (Chi2 $=0.44, \mathrm{dl}=1, \mathrm{p}=0.50)$, l'âge $(\mathrm{t}(84)=0.49, \mathrm{p}=0.62$ ) et le type d'établissement fréquenté (favorisé / défavorisé) (Chi2 =0.19, dl = $1, p=0.66)$. 
Tableau II. Age, niveau de scolarité et type d'établissement fréquenté des élèves interviewés : moyenne (Ecart-Type), nombre d'élèves

\begin{tabular}{|l|l|l|l|l|l|l||}
\hline \multirow{2}{*}{ Sexe } & Total & Age & \multicolumn{2}{|l|}{ Niveau de scolarité } & \multicolumn{2}{|c|}{ Type d'établissement } \\
\cline { 3 - 7 } & & $\mathrm{M}(\mathrm{ET})$ & Collège & Lycée & Favorisé & Défavorisé \\
\hline \multirow{2}{*}{ Filles } & 43 & $\begin{array}{l}16.3 \\
(2.7)\end{array}$ & 18 & 25 & 21 & 22 \\
\hline \multirow{2}{*}{ Garçons } & 43 & $\begin{array}{l}16.0 \\
(2.6)\end{array}$ & 15 & 28 & 19 & 24 \\
\hline \multirow{2}{*}{ TOTAL } & 86 & $\begin{array}{l}16.1 \\
(2.6)\end{array}$ & 33 & 53 & 40 & 46 \\
\hline
\end{tabular}

\section{3. Analyse des données}

Une analyse des entretiens de type "logico-sémantique " a été menée (Mucchielli 1998). Le corpus de chacun des entretiens a été standardisé, c'est-à-dire organisé selon les axes de questionnement. Pour déterminer les catégories d'analyse des réponses relatives à la mixité, la méthode inductive a été utilisée : aucune catégorie de réponses n'a été conçue à l'avance. Les catégories d'analyse retenues figurent dans les tableaux de résultats (cf Tableaux III à V). Les réponses des élèves ont été analysées en général puis en comparant les réponses des filles et des garçons, des collégiens et des lycéens, des élèves d'établissements favorisés et défavorisés. Un indice a été calculé pour pouvoir comparer les réponses des collégiens et lycéens et celles des élèves d'établissements favorisés et défavorisés. Cet indice correspond au nombre de mentions divisé par le nombre d'élèves : on obtient ainsi un nombre moyen de mentions par élève. Pour la variable "sexe ", ce calcul n'est pas nécessaire puisque le même nombre de filles et de garçons a été interviewé.

\section{Résultats}

Les attitudes des élèves du second degré à l'égard de la mixité peuvent se caractériser par quatre éléments : une attitude plutôt favorable à la mixité en EPS ; de nombreux avantages perçus reliés à la présence des garçons en EPS ; des inconvénients plus rares liés prioritairement aux différences de capacités physiques entre les filles et les garçons ; et enfin des mises en doute quant à la mise en place réelle de la mixité.

3. 1. Des élèves favorables à la mixité en EPS 
Tableau III. Pour ou contre la mixité : répartition des réponses des élèves selon leur sexe, leur niveau de scolarité et le type d'établissement fréquenté, nombre d'élèves (\% colonnes arrondis)

\begin{tabular}{|c|c|c|c|c|c|c|c|}
\hline & \multirow[t]{2}{*}{ Total } & \multicolumn{2}{|l|}{ Sexe } & \multicolumn{2}{|c|}{ Niveau de scolarité } & \multicolumn{2}{|c|}{$\begin{array}{l}\text { Type } \\
\text { d'établissement }\end{array}$} \\
\hline & & Filles & Garçons & Collège & Lycée & Favorisé & Défavorisé \\
\hline \multicolumn{8}{|c|}{ Réserves émises par rapport à la mixité effective en EPS } \\
\hline $\begin{array}{l}\text { Nombre } \\
\text { réserves }\end{array}$ & 18 & 13 & 5 & \begin{tabular}{|l}
14 \\
$(0.42) 1$
\end{tabular} & $4(0.07)$ & $4(0.10)$ & $14(0.30)$ \\
\hline \multicolumn{8}{|c|}{ Pour ou contre la mixité } \\
\hline $\begin{array}{l}\text { Nombre } \\
\text { d'élèves }\end{array}$ & 84 & 43 & 41 & 33 & 51 & 38 & 46 \\
\hline Pour la mixité & \begin{tabular}{|ll}
70 & $(83$ \\
$\%)$ &
\end{tabular} & \begin{tabular}{|ll}
36 & $(84$ \\
$\%)$ &
\end{tabular} & $34(83 \%)$ & $28(85 \%)$ & $42(82 \%)$ & $\begin{array}{l}38 \\
(100 \%)\end{array}$ & $32(70 \%)$ \\
\hline $\begin{array}{ll}\text { Contre } & \text { la } \\
\text { mixité } & \end{array}$ & \begin{tabular}{|ll}
9 & $(11$ \\
$\%)$ &
\end{tabular} & \begin{tabular}{|ll}
5 & $(12$ \\
$\%)$ &
\end{tabular} & $4(10 \%)$ & $5(15 \%)$ & $4(8 \%)$ & 0 & $9(20 \%)$ \\
\hline $\begin{array}{l}\text { Dépend } \\
\text { A.P.S.A. }\end{array}$ & $5(6 \%)$ & $2(4 \%)$ & $3(7 \%)$ & 0 & $5(10 \%)$ & 0 & $5(10 \%)$ \\
\hline
\end{tabular}

La majorité des élèves d'établissements secondaires, filles comme garçons, est favorable à la mixité en EPS (83\% d'entre eux) (cf. Tableau III) : «Je pense que ça ne serait vraiment pas intelligent de la part des professeurs, de ne pas mélanger [...] On n'est pas dans les anciennes écoles. Entre garçons ce n'est pas bien ! » (S69*Fav*Ly*G).

La totalité des élèves d'établissements favorisés sont pour la mixité en EPS. Des lycéens d'établissements défavorisés n'arrivent pas à prendre position (6\% d'entre eux) et pensent que ça dépend des activités pratiquées. Selon eux, les sports collectifs surtout devraient être non mixtes : «ça dépend pour quelles activités. Déjà la natation, je préfère être séparée des garçons parce qu'on est en maillot de bain [...] Et même en foot ! Que ce soit foot, basket, pareil ils [les garçons] gardent la balle pour eux » (S40*DéfavLy*F).

3. 2. Des avantages multiples liés à la présence des garçons Les avantages perçus à l'égard de la mixité en EPS sont multiples (99 mentions, 7 types). Les filles tendent à en citer un plus grand nombre que les garçons (56 contre 43 mentions). II en est de même entre les élèves d'établissements favorisés et défavorisés ( 1.40 contre 0.93 mentions / élève) (cf. Tableau IV). 
Tableau IV. Avantages liés à la mixité en EPS : répartition des réponses des élèves selon leur sexe, leur niveau de scolarité et le type d'établissement fréquenté, nombre d'avantages cités (\% colonnes arrondis)

\begin{tabular}{|c|c|c|c|c|c|c|c|}
\hline & \multirow[t]{2}{*}{ Total } & \multicolumn{2}{|l|}{ Sexe } & \multicolumn{2}{|c|}{ Niveau de scolarité } & \multicolumn{2}{|c|}{ Type d'établissement } \\
\hline & & Filles & Garçons & Collège & Lycée & Favorisé & Défavorisé \\
\hline \begin{tabular}{|l} 
Nombre \\
d'avantages
\end{tabular} & 99 & 56 & 43 & $40(1.21) 1$ & $\begin{array}{l}59 \\
(1.11)\end{array}$ & $56(1.40)$ & $43(0.93)$ \\
\hline Ambiance & $\begin{array}{|ll|}59 & (60 \\
\%) & \\
\end{array}$ & $\begin{array}{|ll|}32 & (57 \\
\%) & \\
\end{array}$ & $\begin{array}{|ll|}27 & (63 \\
\%) & \\
\end{array}$ & $21(53 \%)$ & $\begin{array}{l}38 \\
(64 \%)\end{array}$ & $34(61 \%)$ & 25 (58\%) \\
\hline Motivation & $\begin{array}{|ll|}14 & (14 \\
\% & \\
\end{array}$ & $\begin{array}{|ll|}13 & (23 \\
\%) & \end{array}$ & $1(2 \%)$ & $6(15 \%)$ & $8(14 \%)$ & $6(11 \%)$ & $8(19 \%)$ \\
\hline Entraide & $\begin{array}{|ll|}11 & (11 \\
\%) & \end{array}$ & $7(12 \%)$ & $4(9 \%)$ & $8(20 \%)$ & $3(5 \%)$ & $6(11 \%)$ & $5(12 \%)$ \\
\hline \begin{tabular}{|l|} 
Connaître \\
l'autre sexe
\end{tabular} & $7(7 \%)$ & $2(4 \%)$ & $5(12 \%)$ & $3(8 \%)$ & $4(7 \%)$ & $3(5 \%)$ & $4(9 \%)$ \\
\hline \begin{tabular}{|ll} 
Rapport & de \\
séduction & \\
\end{tabular} & $6(6 \%)$ & $1(2 \%)$ & $5(12 \%)$ & $1(2 \%)$ & $5(8 \%)$ & $6(11 \%)$ & 0 \\
\hline Plus équitable & $1(1 \%)$ & $1(2 \%)$ & 0 & 0 & $1(2 \%)$ & $1(1 \%)$ & 0 \\
\hline $\begin{array}{l}\text { Garçons } \\
\text { faibles avec } \\
\text { filles }\end{array}$ & $1(1 \%)$ & 0 & $1(2 \%)$ & $1(2 \%)$ & 0 & 0 & $1(2 \%)$ \\
\hline Avantages liés a & au relatio & nnel ou à & réussite & & & & \\
\hline Relationnel & $\begin{array}{|ll|}72 & (26 \\
\%) & \\
\end{array}$ & $\begin{array}{|ll|}35 & (64 \\
\%) & \\
\end{array}$ & \begin{tabular}{|ll}
37 & $(86$ \\
$\%)$ & \\
\end{tabular} & $25(63 \%)$ & $\begin{array}{l}47 \\
(81 \%)\end{array}$ & $43(77 \%)$ & $29(67 \%)$ \\
\hline Réussite & $\begin{array}{|ll|}26 & (74 \\
\% & \\
\end{array}$ & $\begin{array}{|ll|}20 & (36 \\
\%) & \\
\end{array}$ & $6(14 \%)$ & $15(37 \%)$ & $\begin{array}{l}11 \\
(19 \%)\end{array}$ & $12(23 \%)$ & $14(33 \%)$ \\
\hline
\end{tabular}

Les principaux avantages cités font tous référence à des qualités des garçons et de façon explicite ou implicite à des défauts des filles :

- Des garçons « déconneurs », des filles « chiantes » : La mixité apporte prioritairement un bénéfice au niveau de l'ambiance de classe (60\% des avantages cités). Les garçons mettent l'ambiance, sont plus déconneurs et marrants. Les filles discutent beaucoup et 
font des histoires pour rien : « C'est vrai que les garçons sont un peu plus déconneurs que les filles. Donc ça fait une ambiance plus décontractée en sport, ça fait moins cours de math[ématique]s » $\left(S 63^{\star} F a v^{\star} L y^{\star} F\right)$.

- Des garçons « motivants, dynamiques », des filles « molles, démotivantes »: La mixité apporte sur le plan motivationnel (14\% des avantages cités). Les garçons ont un meilleur niveau et c'est plus motivant, ça bouge plus avec les garçons. Les filles sont peu motivées et c'est plus mou : « Les garçons, ils nous poussent un peu parce que sinon on ne fait rien pendant le match. Je ne sais pas, ça nous motive » (S19*Défav*Co*F).

- Des garçons «forts », des filles « faibles » : La mixité permet de s'entraider (11\% des mentions). Les garçons savent plus de choses et peuvent aider les filles : « Les garçons, ils ont un bon niveau en sport, ils peuvent nous donner des conseils, et on peut progresser »(S30*Fav*Co*F).

- Apprendre à connaître l'autre sexe : La mixité permet également de créer des liens entre les deux sexes, de mieux connaître l'autre sexe, d'évaluer et de bénéficier des qualités de chacun (7\% des mentions) : «On voit les différences » (S27*Fav*Co*G).

- Séduire l'autre sexe : La mixité permet également de séduire, elle favorise le contact entre les deux sexes (6\% des mentions) : « [...] ça perturbe un peu, il faudrait leur mettre des foulards [...] À la piscine, les mettre dans des combinés, avec un masque bien moche » (S64*Défav*Ly*G).

« L'ambiance de classe » est l'avantage le plus fréquemment cité par les filles et les garçons (57\% et $63 \%$ des mentions). Sinon, les avantages liés à la mixité ont tendance à différer selon le sexe des élèves. Le fait de « connaître l'autre sexe » et le « rapport de séduction » sont des avantages cités surtout par des garçons (12\% des mentions chez les garçons, $4 \%$ et $2 \%$ chez les filles). Les filles, quant à elles, citent prioritairement la « motivation » procurée par la présence des garçons (23\% et $2 \%$ des mentions) ainsi que la «possibilité d'entraide entre pairs » (12\% et $9 \%$ des mentions).

De moindres différences au niveau des avantages cités sont observables selon le niveau de scolarité des élèves et le type d'établissement fréquenté. Notons, tout de même, que « la possibilité d'entraide » tend à être citée plus fréquemment par les collégiens (20\% et $5 \%$ des mentions). Les lycéens, quant à eux, citent plus fréquemment le « rapport de séduction » comme avantage relié à la mixité ( $8 \%$ et $2 \%$ des mentions). Cet avantage est seulement cité par des élèves d'établissements favorisés (11\% des mentions) (cf. Tableau IV).

Les avantages ont été classés en deux catégories : des avantages reliés à la réussite en EPS (motivant, entraide, pratique avec élèves de même niveau) et des avantages au plan 
relationnel non reliés à la réussite (ambiance, connaître l'autre et le rapport de séduction). Les élèves citent prioritairement des apports de la mixité au niveau relationnel (74\% des mentions). Cependant, les filles, tendent à percevoir davantage d'apports au niveau de la réussite que les garçons (36\% et $14 \%$ des mentions). II en est de même entre les collégiens et les lycéens (37\% et $19 \%$ des mentions) et les élèves d'établissements défavorisés et favorisés (33\% et $23 \%$ des mentions) (cf. Tableau 4).

3. 3. Des inconvénients plus rares liés aux différences intersexes de capacités physiques

Tableau V. Inconvénients liés à la mixité : répartition des réponses des élèves selon leur sexe, leur niveau de scolarité et le type d'établissement fréquenté, nombre d'inconvénients cités ( $\%$ colonnes arrondis)

\begin{tabular}{|c|c|c|c|c|c|c|c|}
\hline & \multirow[t]{2}{*}{ Total } & \multicolumn{2}{|l|}{ Sexe } & \multicolumn{2}{|c|}{$\begin{array}{ll}\text { Niveau } & \text { de } \\
\text { scolarité } & \end{array}$} & \multicolumn{2}{|c|}{$\begin{array}{l}\text { Type } \\
\text { d'établissement }\end{array}$} \\
\hline & & Filles & Garçons & Collège & Lycée & Favorisé & Défavorisé \\
\hline $\begin{array}{l}\text { Nombre } \\
\text { d'inconvénients }\end{array}$ & 21 & 12 & 9 & $\begin{array}{l}11 \\
(0.33) 1\end{array}$ & $\begin{array}{l}10 \\
(0.19)\end{array}$ & $4(0.10)$ & $17(0.37)$ \\
\hline $\begin{array}{l}\text { Garçons jouent } \\
\text { entre eux }\end{array}$ & $\begin{array}{|ll|}11 & (53 \\
\%) & \\
\end{array}$ & $\begin{array}{ll}11 & (92 \\
\%) & \end{array}$ & 0 & $6(55 \%)$ & $\begin{array}{l}5 \\
(50 \%)\end{array}$ & $\begin{array}{l}4 \\
(100 \%)\end{array}$ & $7(41 \%)$ \\
\hline Filles $=$ handicap & $\begin{array}{|ll|}7 & (33 \\
\%) & \\
\end{array}$ & 0 & $7(78 \%)$ & $2(18 \%)$ & $\begin{array}{l}5 \\
(50 \%)\end{array}$ & 0 & $7(41 \%)$ \\
\hline Aucune entraide & $\begin{array}{|ll|}3 & (14 \\
\%) & \\
\end{array}$ & $1(8 \%)$ & $2(22 \%)$ & $3(27 \%)$ & 0 & 0 & $3(18 \%)$ \\
\hline
\end{tabular}

Les élèves perçoivent moins d'inconvénients que d'avantages liés à la mixité en nombre (21 mentions) et en diversité (3 types). Ces inconvénients tendent à être plus fréquemment cités par des filles (12 contre 9 mentions), des collégiens ( 0.33 contre 0.195 ) et des élèves d'établissements défavorisés (0.37 contre 0.105) (cf. Tableau 5).

Les deux principaux inconvénients font référence aux différences intersexes de niveau en situation de jeu :

- Des garçons trop individualistes dans le jeu : Les élèves se plaignent majoritairement du comportement trop individualiste des garçons en situation de jeu (53\% des inconvénients cités). Les filles sont comme « invisibles » lorsqu'elles jouent avec les garçons : « C'est assez difficile de faire des bonnes actions avec eux [les garçons], étant donné qu'ils jouent tout seul » (S32*FavCo*F). 
- La présence des filles, un handicap en EPS : Des élèves perçoivent la présence des filles comme un « handicap » car elles sont trop faibles et elles font baisser la note (33\% des mentions) : «Oui ça ralentit le jeu [...]. La fille, elle va rester avec le ballon, alors que nous [les garçons], si on a le ballon, on dribble et on marque. Voilà, on est plus fort ! » (S11* Défav $\left.{ }^{*} \mathrm{Co}^{*} \mathrm{G}\right)$.

- Aucune entraide intersexe : Enfin, pour certains, la mixité est inutile puisqu'il n'y a pas d'entraide entre élèves de sexe opposé ( $14 \%$ des mentions) : « Je préfère être qu'avec les filles. Ils [les garçons] ne nous aident pas trop en réalité 》 (S14*Défav $\left.{ }^{*} \mathrm{Co}^{*} \mathrm{~F}\right)$.

Comme les avantages, les inconvénients reliés à la mixité diffèrent selon le sexe des élèves. Seules des filles se plaignent du fait que les garçons sont " trop individualistes " en situation de jeu (92\% de leurs mentions). Seuls des garçons perçoivent les filles comme un «handicap " en situation de mixité en EPS (78\% de leurs mentions) (cf. Tableau V).

Des différences sont également observables selon le niveau de scolarité des élèves et le type d'établissement fréquenté. Seuls les collégiens citent le manque d'entraide ( $27 \%$ des mentions). Et les élèves d'établissements favorisés ne perçoivent qu'un seul inconvénient : le comportement trop individualiste des garçons (cf. Tableau 5).

3. 4. Une mise en doute de la mixité réelle en EPS

Certains élèves disent être toujours ou très souvent séparés des élèves de sexe opposé et déplorent ceci. Ces réserves, quant à la mise en place effective de la mixité en EPS, tendent à être surtout émises par des élèves de sexe féminin (13 contre 5 mentions), de collège $(0.42$ contre 0.075$)$ et d'établissements défavorisés $(0.30$ contre 0.105$)$ (cf. Tableau 3). Deux éléments sont mis en cause : la constitution de groupes non mixtes par l'enseignant lui-même et la constitution des groupes par les élèves qui aboutit le plus souvent à des équipes non mixtes : « En fait ce n'est pas mixte, il [l'enseignant] sépare les filles des garçons ou il dit : «Choisissez vos équipes !» » $\left(S 2^{\star} \operatorname{Défav}^{*} \mathrm{Co}^{*} \mathrm{G}\right)$.

L'activité la plus fréquemment citée comme non mixte est le football : « Genre en foot[ball], il [l'enseignant] nous sépare. Les filles sont d'un côté et les garçons sont d'un autre côté. Ce n'est pas normal [...] Ce n'est pas parce qu'on ne sait pas trop jouer qu'on ne peut pas apprendre avec les garçons qui savent plus de choses, qui pourraient nous montrer plus de choses » (S1*Défav*Co*F).

\section{Discussion}

Les élèves du second degré sont plutôt satisfaits de la mixité en EPS. Ils sont majoritairement pour la mixité et perçoivent plus d'avantages que d'inconvénients à sa 
mise en place. Ces résultats confirment notre hypothèse et les résultats des études antérieures. Dans l'étude de Dallaire et Rail (1996), 79 \% des élèves interrogés sont pour la mixité, ils sont $83 \%$ dans notre étude.

Certains élèves ont des difficultés à prendre position sur la question de la mixité en EPS vue la diversité des activités pratiquées: individuelles ou collectives, aquatiques ou terrestres, connotées féminines, neutres ou masculines, etc. Ces élèves pensent que certaines activités doivent être mixtes et d'autres non. Les sports collectifs sont les plus cités comme devant être non mixtes: les différences de niveau de jeu en sont les principales causes. Le port de maillot de bain en natation est également une source de mal-être face au regard des élèves de sexe opposé. Ces propos montrent toute la complexité à réfléchir au problème de la mixité en EPS.

Des élèves mettent en doute la mise en place effective de la mixité en EPS. Ceci reflète d'une certaine manière la réalité. Si les programmes tentent d'évacuer le sexisme des pratiques sportives en offrant la possibilité d'un enseignement égalitaire, de leur côté les enseignants, par conviction ou par habitude, règlent différemment ce problème. Ils proposent souvent une mixité superficielle où le contact à l'autre sexe est évité (Artus 1999). Globalement, l'enseignement en mixité décline de la 6 e (95\% de classes) à la terminale $(60 \%)$ : une rupture importante se situe entre le collège et le lycée (3e : $84 \%$, 2 e : $67 \%$ ). La mixité en EPS est moins fréquente dans les établissements en Zone d'Education Prioritaire et dans les lycées professionnels. Sa mise en place effective diffère également d'une activité à l'autre (Terret, Cogérino \& Rogowsky 2006).

Comme supposé, les collégiens et les élèves d'établissements défavorisés ont une attitude plus négative à l'égard de la mixité que les lycéens et les élèves d'établissements favorisés. Seuls des élèves d'établissements défavorisés sont contre la mixité et ces élèves citent moins d'avantages reliés à la mixité que ceux d'établissements favorisés. Les collégiens, comme les élèves d'établissements défavorisés, citent plus d'inconvénients à l'égard de la mixité et davantage de réserves quant à sa mise en place effective. Ces résultats peuvent être reliés à la plus grande difficulté d'aller vers l'autre sexe au collège qu'au lycée et à l'adhésion aux stéréotypes de sexe plus rigide dans les milieux défavorisés (Bouchard \& Saint-Amand 1996 ; Passeron \& De Singly 1984). Les collégiens sont en pleine phase pubertaire et en quête d'une identité sexuée ; ils cherchent à se démarquer de l'autre sexe. Durant cette période, les normes de genre sont très rigides et les comportements très stéréotypés (Alfieri, Ruble \& Higgins 1996 ; Braconnier 1995 ; Galambos et al. 1990). 
Contrairement à d'autres travaux et à l'hypothèse émise, cette étude ne permet pas de conclure à une attitude plus favorable des garçons à l'égard de la mixité. Les réponses des filles et des garçons sont ambiguës. Les filles sont aussi nombreuses que les garçons à être pour la mixité en EPS. Elles citent un plus grand nombre d'avantages mais également un plus grand nombre d'inconvénients reliés à la mixité. La supériorité des garçons est perçue par les filles du second degré comme un problème mais également comme un avantage. Cette ambiguïté se retrouve également dans d'autres travaux (Garrett 2004 ; Warrington \& Younger 2000 ; Wright 1996).

La mixité est perçue positivement grâce à la présence des garçons : les garçons « mettent l'ambiance », sont «plus motivants », sont «plus forts » et peuvent aider les filles. Les garçons sont perçus par les pairs comme plus marrants en milieu scolaire (Kehily \& Nayak 1997 ; Lahelma 2002) : leurs blagues et leur manque de maturité permettent de réduire la lassitude du quotidien des cours. Les perceptions chez les élèves de leur propre sexe sont en lien avec le discours sur l'hégémonie masculine et les stéréotypes de féminité et de masculinité (Garett 2004 ; MacDonald 1990 ; Wright 1996). Les filles comme les garçons opposent les qualités de compétence des garçons à la faiblesse des filles. En EPS, élèves et enseignants sont complices pour maintenir les filles comme inférieures aux garçons.

Les apports de la mixité au niveau relationnel sont très importants pour les élèves. La priorité des élèves en EPS ne semble pas la réussite mais la détente, le défoulement, l'ambiance de classe: c'est la seule discipline scolaire où ils peuvent se dépenser physiquement et avoir des relations plus directes avec les camarades de classe. Ces dernières leur permettent de mieux connaître l'autre sexe (ses capacités, désirs, façons de penser et d'agir), voire de le séduire. Pour répondre au mieux aux attentes des élèves et pour que l'EPS reste la discipline préférée des élèves (Terrail 1992), il semble important que l'EPS ne « s'intellectualise » pas trop (fiches à remplir, projet à construire) et conserve ce côté « détente » dans sa quête d'identité en tant que discipline d'enseignement.

Les apports de la mixité au niveau de la réussite sont davantage cités par des filles, des collégiens et des élèves d'établissements défavorisés. Par ailleurs, seuls des élèves d'établissements défavorisés et des collégiens citent le manque d'entraide comme inconvénient. Ces élèves semblent avoir plus à gagner avec la mixité. Les filles, les élèves d'établissements défavorisés et de collèges sont moins sportifs que les garçons, les élèves d'établissements favorisés et de lycées (Choquet \& Ledoux 1994 ; Muller 2003). Wright (1996) a également montré que les préférences pour la classe mixte chez les filles étaient reliées au niveau supérieur des garçons, les aidant à apprendre plus vite. Les garçons apprécient la mixité surtout pour des aspects non reliés à la réussite (Dallaire \& 
Rail 1996 ; Gagné \& al. 2006) : elle permet, selon eux, de connaître les filles, de développer des relations personnelles, de se sentir supérieur, d'aider les filles et de faire son « show".

Si les filles reconnaissent des qualités chez les garçons (force, motivation, humour), les garçons se plaignent de la présence des filles qui atténuent la qualité de leur jeu. Pour les garçons, les filles semblent les bienvenues surtout lorsqu'elles les encouragent, les admirent ou flirtent avec eux ; mais lorsqu'il s'agit de pratiquer réellement du sport, elles sont perçues comme un « handicap ». Ces résultats rejoignent ceux de Wright (1996) ou d'Artus (1999). Le manque d'habileté des filles, leur manque de motivation et la modification du jeu que les garçons sont capables de développer seuls posent problème aux garçons (Wright 1996). Les filles sont d'autant plus appréciées par les garçons en EPS si elles sont compétentes et actives. Selon l'étude d'Artus (1999), les garçons de lycée sont plus nombreux à penser que les filles sont un frein à l'engagement physique (27\% contre $17 \%)$.

La dichotomie du féminin et masculin (relationnel / domination) se retrouve dans les dires des élèves. Les garçons apportent aux filles leurs savoirs, compétences dans le domaine du sport et permettent de les faire progresser en EPS, alors que les filles apportent aux garçons seulement au niveau relationnel (liens affectifs ou amoureux). Souvent, les stéréotypes de sexe comportent un fond de vérité mais le problème réside dans la généralisation de ce fond de vérité à l'ensemble des membres du groupe auquel il est appliqué (Leyens, Yzerbyt \& Schradron 1996). Par exemple, beaucoup de garçons sont forts en EPS mais tous ne le sont pas ... et certaines filles le sont (Vigneron 2004 ; Lentillon 2006).

On retrouve également une valorisation du masculin en EPS dans les dires des élèves, comme ceci a été observé dans les activités et contenus enseignés en EPS ou dans les attentes et interventions de l'enseignant (Cogérino 2005 ; Couchot-Schiex 2005 ; Flintoff 1994 ; Skelton 2002 ; Vigneron 2004). Les caractéristiques masculines (force, motivation, humour) sont perçues positivement par les élèves et sont celles recherchées en EPS. L'EPS est reconnue par les élèves comme un lieu masculin, où le pouvoir revient aux garçons et le rôle des filles semble réduit à les féliciter, à rire à leurs blagues et à rester invisibles dans la pratique. Les élèves ne semblent pas prêts au changement: la domination des garçons en EPS est la norme pour les élèves. Ceci a bien été mis en évidence dans l'analyse plus large des injustices perçues chez les élèves en EPS (Lentillon \& Cogérino 2005 ; Lentillon 2006).

5. Conclusion 
Les élèves du second degré sont globalement favorables à la mixité en EPS, surtout les lycéens et les élèves d'établissements favorisés. Les attitudes des filles et des garçons sont ambiguës et très stéréotypées à l'égard de la mixité. Les garçons, perçus comme forts, sont une source d'aide en EPS mais leurs capacités physiques supérieures sont également perçues comme un obstacle en situation de mixité. Les filles, perçues comme faibles, sont appréciées en EPS seulement lorsqu'elles félicitent, encouragent les garçons; par contre lorsqu'il s'agit de pratiquer, leur faible niveau reste problématique. Comme les enseignants, les élèves valorisent les qualités masculines en EPS et cette intériorisation de la domination masculine chez les élèves contribue inévitablement à la persistance des inégalités intersexes en EPS.

\section{Bibliographie}

Alfieri, T., Ruble, D.N., \& Higgins, E.T. (1996). Gender stereotypes during adolescence: Developmental changes and the transition to junior high school. Developmental Psychology, 32(6), 1129-1137.

Arnaud, P., \& Terret, T. (1996). Histoire du sport féminin. Paris : L'Harmattan.

Artus, D. (1999). La mixité : illusion égalitaire en éducation physique et sportive ? Son étude à travers les représentations sociales des enseignants et des lycéens. Thèse de doctorat non publiée, Université de Poitiers.

Bouchard, P., \& Saint-Amant, J.-C. (1996). Garçons et filles, stéréotypes et réussite scolaire. Montréal : Editions du remue-ménage.

Braconnier, A. (1995). Adolescentes, adolescents. Psychopathologie différentielle. Paris: Bayard Editions.

Choquet, M., \& Ledoux, S. (1994). Adolescents, enquête nationale. Paris: Edition INSERM.

Cleuziou, J.-P. (2000). L'analyse des menus et des notes. In B. David (ed.), Education physique et sportive : La certification au baccalauréat (pp. 77-124). Paris : INRP.

Coelho, J.D. (2000). Student perceptions of physical education in a mandatory college program. Journal of Teaching in Physical Education, 19(6), 1-24.

Cogérino, G. (2005). Filles et garçons en EPS. Paris : Ed. Revue EPS.

Colwell, S. (1999). Feminism and figurational sociology: contributions to understandings of sports, physical education and sex/gender. European Physical Education Review, 5(3), 219-240. 
Combaz, G. (1991). La mixité en EPS : opinions et souhaits des élèves. Revue EP.S, 231, 62-65.

Connell, R.W. (1995). Masculinities. Cambridge, UK : Polity Press.

Couchot-Schiex, S. (2005). Contributions aux effets du genre de l'enseignant. Étude descriptive dans trois APSA : gymnastique, badminton, hand-ball. Thèse doctorale non publiée, Université de Sciences sociales, Lyon 2.

Couchot-Schiex, S., \& Trottin, B. (2005). Interactions enseignants-élèves en EPS : variations en fonction du sexe et du genre. In G. Cogérino (ed.), Filles et garçons en EPS (pp. 163-179). Paris : Ed. Revue EPS.

Dallaire, H., \& Rail, G. (1996). Vers l'équité en EPS. Canada : Bibliothèque nationale du Canada, Réseau National d'Action Education Femme (RNAEF).

Duru-Bellat, M. (2002). Les inégalités scolaires. Genèse et mythes. Paris : PUF, Education et formation.

Flintoff, A. (1994). Sexism and homophobia in physical education: the challenge for teacher educators. Physical Education Review, 17(2), 97-105.

Gagné, G., Martel, D., \& Nadeau, L. (2006). Raisons évoquées par des élèves pour justifier leur préférence en matière de mixité ou de non mixité en éducation physique. In G. Carlier, D. Bouthier, \& G. Bui-Xuân (Eds.), Intervenir en éducation physique et en sport : recherches actuelles (pp. 378-385). Belgique : PUL.

Galambos, N.L., Almeida, D.M., \& Petersen, A.C. (1990). Masculinity, femininity, and sex role attitudes in early adolescence: Exploring gender intensification. Child Development, 61(6), 1905-1914.

Garrett, R. (2004). Negotiating a physical identity: girls, bodies and physical education. Sport, Education \& Society, 9(2), 223-237.

Gilroy, S. (1989). The embodiment of power: gender and physical activity. Leisure Studies, 8(2), 163-172.

Griffin, P.S. (1984). Girls' participation in a middle school team sports unit. Journal of Teaching in Physical Education, 4, 30-38.

Kehily, M.J., \& Nayak, A. (1997). Lads and laughter: humour and the production of heterosexual hierarchies. Gender and Education, 9(1), 69-87.

Lahelma, E. (2002). Gendered conflicts in secondary school: fun or enactment of power ?. Gender and Education, vol. 14(3/1), 295-306.

Lentillon, V. (2006). Les inégalités intersexes en EPS : injustices perçues chez les élèves à l'égard des notes, des interventions de l'enseignant et des interactions 
entre pairs. Thèse doctorale en STAPS non publiée, Université Claude Bernard Lyon 1.

Lentillon, V., \& Cogérino, G. (2005). Les inégalités entre les sexes dans l'évaluation en EPS : sentiment d'injustice chez les collégiens. Revue STAPS, 68, 79-95.

Lentillon, V., \& Trottin, B. (2005). Relations Educatives en Education Physique et Sportive et perceptions chez des collégiennes et collégiens Français. Revue Education et Francophonie, 33(1), 57-72: http://www.acelf.ca.

Leroy, F., Rousseau, E., Carlier, G., \& Renard, J.-P. (1998). Interventions pédagogiques et incidents disciplinaires dans le cadre des cours d'EP. Actes du Colloque AFRAPS -EDPM : identifier les effets de l'intervention en motricité humaine, CD-Rom, Université Catholique de Louvain, Louvain la neuve, 13-14 mars.

Leyens, J.-P., Yzerbyt, V., \& Schadron, G. (1996). Stéréotypes et cognition sociale. Liège : Mardaga.

MacDonald, D. (1990). The relationship between the sex composition of physical education classes and teacher-pupil verbal interaction. Journal of Teaching in Physical Education, 9, 152-163.

Mosconi, N. (1994). Femmes et savoir. La société, l'école et la division sexuelle des savoirs. Paris : L'Harmattan.

Mucchielli, R. (1998). L'analyse de contenu : documents et des communications. Paris : Ed. ESF, collection « formation permanente », séminaire Mucchielli.

Muller, L. (2003). La pratique sportive des jeunes dépend avant tout de leur milieu socioculturel. Stat-info, 03-07, novembre : http://www.jeunessesports.gouv.fr/ministre_68.html.

Naess, F.J. (2001). Narratives about young men and masculinities in organised sport in Norway. Sport, Education \& Society, 6(2), 125-142

Passeron, J.-C., \& De Singly, F. (1984). Différences dans la différence : socialisation de classe et socialisation sexuelle. Revue Française de Science Politique, 34(1), 4878.

Rønholt, H. (2002). 'It's only the sissies ...': analysis of teaching and learning processes in physical education: a contribution to the hidden curriculum. Sport, Education \& Society, 7(1), 25-36.

Satina, B., Solmon, M.A., Cothran, D.J., Loftus, S.J., \& Stocking-Davidson, K. (1998). Patriarchal consciousness: middle school students' and teachers' perspectives of motivational practices. Sport, Education \& Society, 3, 181-200. 
Skelton, C. (2002). Constructing dominant masculinity and negotiating the "male gaze". International Journal of Inclusive Education, 6(1), 17-31.

Terrail, J.-P. (1992). Parents, filles et garçons, face à l'enjeu scolaire. Education et Formations, 30, 3-11.

Terret, T. (2004). Sport et masculinité : une revue de questions. Revue STAPS, 66, 209225.

Terret, T., Cogérino, G., \& Rogowski, I. (2006). Pratiques et représentations de la mixité en EPS. Marseille : co-ed. AFRAPS - Revue EPS.

Vertinsky, P.A. (1992). Reclaiming space, revisioning the body: the quest for a gender sensitive physical education. Quest, 44, 373-396.

Vigneron, C. (2004). La construction des inégalités de réussite en EPS au baccalauréat entre filles et garçons. Thèse doctorale en Sciences de l'éducation non publiée, Université de Dijon.

Warrington, M., \& Younger, M. (2000). The other side of the gender gap. Gender and Education, 12(4), 493-508.

Wright, J.M. (1996). The construction of complementarity in physical education. Gender and Education, 8(1), 61-79.

Zaidman, C. (1995). École, mixité, politique de la différence des sexes. In M. De Manassein (ed.), De l'égalité des sexes (pp. 219-232). Paris : CNDP, Documents, actes et/pour l'éducation. 